\title{
Abstractive Text Summarization with Hierarchical Multi-scale Abstraction Modeling and Dynamic Memory
}

\author{
Lihan Wang ${ }^{1,2}$, Min Yang ${ }^{1 \dagger}$, Chengming $\mathrm{Li}^{1 \dagger}$, Ying Shen ${ }^{3}$, Ruifeng $\mathrm{Xu}^{4}$ \\ ${ }^{1}$ Shenzhen Institutes of Advanced Technology, Chinese Academy of Sciences \\ ${ }^{2}$ University of Chinese Academy of Sciences \\ ${ }^{3}$ Sun Yat-Sen University $\quad{ }^{4}$ Harbin Institute of Technology (Shenzhen) \\ \{lh.wang1,min.yang,cm.li\}@siat.ac.cn,sheny76@mail.sysu.edu.cn,xuruifeng@hit.edu.cn
}

\begin{abstract}
In this paper, we propose a novel abstractive text summarization method with hierarchical multi-scale abstraction modeling and dynamic memory (called MADY). First, we propose a hierarchical multi-scale abstraction modeling method to capture the temporal dependencies of the document from multiple hierarchical levels of abstraction, which mimics the process of how human beings comprehend an article by learning fine timescales for low-level abstraction layers and coarse timescales for high-level abstraction layers. By applying this adaptive updating mechanism, the highlevel abstraction layers are updated less frequently and expected to remember the long-term dependency better than the low-level abstraction layer. Second, we propose a dynamic key-value memoryaugmented attention network to keep track of the attention history and comprehensive context information for the salient facets within the input document. In this way, our model can avoid generating repetitive words and faultiness summaries. Extensive experiments on two widely-used datasets demonstrate the effectiveness of the proposed MADY model in terms of both automatic evaluation and human evaluation. For reproducibility, we submit the code and data at: https://github.com/siat-nlp/MADY.git.
\end{abstract}

\section{CCS CONCEPTS}

- Applied Computing $\rightarrow$ Document Management and Text Processing.

\section{KEYWORDS}

Abstractive text summarization, multi-scale abstraction modeling, dynamic memory network

\section{ACM Reference Format:}

Lihan Wang ${ }^{1,2}$, Min Yang ${ }^{1 \dagger}$, Chengming $\mathrm{Li}^{1 \dagger}$, Ying Shen ${ }^{3}$, Ruifeng $\mathrm{Xu}^{4}$. 2021. Abstractive Text Summarization with Hierarchical Multi-scale Abstraction Modeling and Dynamic Memory. In Proceedings of the 44th International ACM SIGIR Conference on Research and Development in Information Retrieval

\footnotetext{
${ }^{\dagger}$ Corresponding authors.

Permission to make digital or hard copies of all or part of this work for personal or classroom use is granted without fee provided that copies are not made or distributed for profit or commercial advantage and that copies bear this notice and the full citation on the first page. Copyrights for components of this work owned by others than ACM must be honored. Abstracting with credit is permitted. To copy otherwise, or republish, to post on servers or to redistribute to lists, requires prior specific permission and/or a fee. Request permissions from permissions@acm.org.

SIGIR '21, July 11-15, 2021, Virtual Event, Canada

(C) 2021 Association for Computing Machinery.

ACM ISBN 978-1-4503-8037-9/21/07 . \$ \$15.00

https://doi.org/10.1145/3404835.3462998
}

(SIGIR '21), July 11-15, 2021, Virtual Event, Canada. ACM, New York, NY, USA, 5 pages. https://doi.org/10.1145/3404835.3462998

\section{INTRODUCTION}

Abstractive text summarization aims to generate condensed and concise summaries that retain the salient information and overall meaning of the source articles. As opposed to the extractive text summarization, which extracts the best summarizing components from the input documents, abstractive summaries potentially contain new phrases and sentences that do not appear in the source documents. Abstractive text summarization has attracted increasing attention recently due to its broad applications in natural language processing (NLP) and information retrieval (IR). In recent years, sequence-to-sequence (seq2seq) models have dominated the study of abstractive text summarization $[9,15,16,18,19]$. The general idea behind these methods is to employ a long short-term memory (LSTM) network [7] to obtain fixed-length sentence representations, and then generates summaries with another LSTM decoder and attention mechanisms.

Although great efforts have been devoted to the abstractive text summarization $[1,10,19,21]$, generating accurate, concise, informative summaries remains a challenge in practice for two primary reasons. First, an article, especially a long document, is usually composed of multiple facets discussed at hierarchical levels of abstraction [20]. To comprehend the hierarchical multi-facets information within the article, it requires hierarchical multi-scale abstraction mining to encode the temporal dependencies with different timescales, which is not exploited in the previous methods. Second, conventional attention mechanisms used in sequence-to-sequence models (seq2seq) are incapable of effectively keeping track of the attention history in learning the dynamic alignment between the neural representations of the source article and the corresponding summary [12]. We argue that lacking comprehensive information (attention history) might result in two issues for abstractive text summarization: (i) generating puzzling words where some subtopics are unnecessarily accessed for multiple times and (ii) generating faultiness summary in which some salient information is mistakenly unexplored.

To alleviate the aforementioned challenges, in this study, we propose a novel MADY method for abstractive text summarization with hierarchical multi-scale abstraction modeling and dynamic memory. MADY improves both the encoding and decoding steps of the seq2seq model by studying how human beings comprehend documents with hierarchical levels of abstraction and write summaries based the dynamic working memory. In encoding, we propose 
a hierarchical multi-scale abstraction modeling (HMAM) model to capture multiple hierarchical levels of abstraction of the source document. Specifically, we encode the temporal dependencies with different timescales, motivated by the fact that the high-level abstraction changes slowly while the low-level abstraction has quickly changing features. In decoding, we propose a dynamic key-value memory-augmented attention (DMA) to alleviate the problem of generating repetitive words and incomplete summary, which allows the model to track the comprehensive information typically for each salient facet within the source document.

We summarize our main contributions as follows. (1) We propose a hierarchical multi-scale pattern mining method to capture the temporal dependencies of the document from multiple hierarchical levels of abstraction. (2) We employ a dynamic key-value memory-augmented attention mechanism to better keep track of attention history and salient information coverage, facilitating the decoder to overcome the problems of generating repetitive and faultiness summary by automatically distinguishing which salient facets have been described and which salient facets are unexplored. (3) Experiments on two benchmark datasets demonstrate that the proposed MADY method outperforms the strong competitors by a substantial margin in terms of both automatic evaluation and human evaluation.

\section{OUR METHODOLOGY}

We assume that each source article $X=\left\{x_{1}, x_{2}, \ldots, x_{n}\right\}$ has a reference summary $Y=\left\{y_{1}, y_{2}, \ldots, y_{k}\right\}$, where $n$ and $k$ denote the length of the source document and reference summary, respectively. Abstractive text summarization aims to generate a summary $\hat{Y}=$ $\left\{\hat{y}_{1}, \hat{y}_{2}, \ldots, \hat{y}_{m}\right\}$ for the source article $X$, where $m$ denotes the length of the generated summary.

As illustrated in Figure 1, the proposed MADY model consists of three primary components: (i) a document encoder that leverages a hierarchical multi-scale recurrent model to learn multiple hierarchical levels of abstraction of the source document; (ii) a dynamic key-value memory-augmented attention module that keeps track of the attention history and encourages the decoder to consider the unexplored salient facets; (iii) a summary decoder that adopts an LSTM decoder to generate the summary word by word. Next, we will introduce each component of MADY in detail.

\subsection{Document Encoder}

2.1.1 Embedding Layer and LSTM Layer. Each word $x_{j}$ in the source document is first converted into a distributed representation $\mathrm{v}_{j}^{e} \in \mathbb{R}^{d_{e}}$ by an embedding layer, where $d_{e}$ denotes the dimension of word embedding. The learned word vectors are then fed into a word-level bidirectional LSTM [15] to learn the hidden states of the input tokens, denoted as $\mathrm{h}^{e}=\left\{\mathrm{h}_{1}^{e}, \mathrm{~h}_{2}^{e}, \ldots, \mathrm{h}_{n}^{e}\right\}$ where $n$ indicates the article length and $\mathrm{h}_{i}^{e}$ is the concatenation of the forward hidden state $\overrightarrow{\mathrm{h}_{i}^{e}}$ and the backward hidden state $\overleftarrow{\mathrm{h}_{i}^{e}}$ at time step $i$ via a simple feed-forward network:

$$
\mathrm{h}_{i}^{e}=\operatorname{ReLU}\left(W_{1}\left[\overrightarrow{\mathrm{h}_{i}^{e}}, \overleftarrow{\mathrm{h}_{i}^{e}}\right]+\mathrm{b}_{1}\right)
$$

where $W_{1}$ is a projection parameter and $b_{1}$ is a bias term.

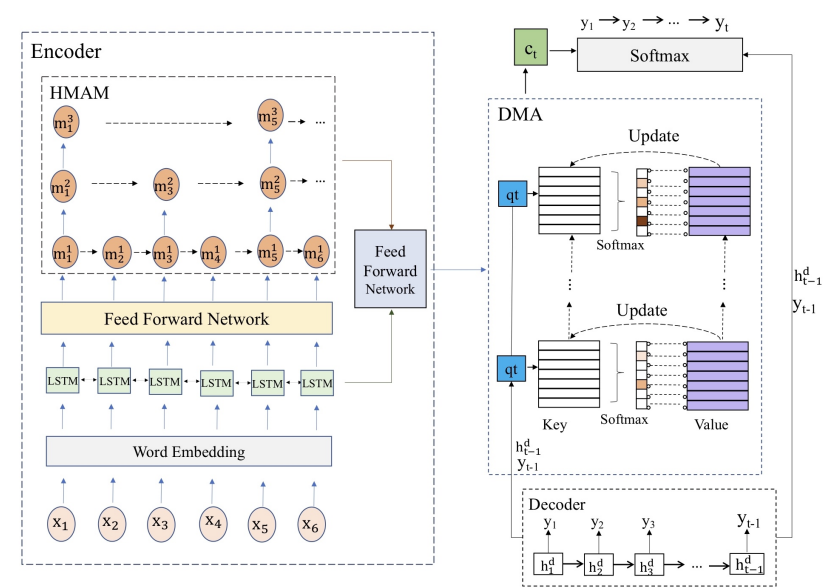

Figure 1: Overview of our MADY model, containing a hierarchical multi-scale abstraction modeling module (HMAM) on the left and a dynamic key-value memory-augmented attention network (DMA) on the right.

2.1.2 Hierarchical Multi-scale Abstraction Modeling. Motivated by how humans summarize articles, we propose a hierarchical multi-scale abstraction modeling (HMAM) method to capture the temporal dependencies within the document from multiple hierarchical levels of abstraction, inspired by [17]. HMAM is a layer-wise hierarchical recurrent memory network containing $D$ layers. Its update rules is similar to the long short-term memory (LSTM) network [7]. HMAM learns fine timescales for low-level abstraction layers and coarse timescales for high-level abstraction layers. By setting different updating timescales as hyperparameters for each layer, the high-level abstraction layers are updated less frequently and expected to remember the long-term dependency better than the low-level abstraction layers.

As shown in Figure 1, we assume that each layer $j$ in HMAM maintains specific memory slots $\mathrm{m}^{j}$. The memory slot $\mathrm{m}_{i}^{j}$ at the $i$-th time step will be transmitted not only to the next time step, but also to the next layer at specific time step. The sequential hidden states $\mathrm{h}^{e}=\left\{\mathrm{h}_{1}^{e}, \mathrm{~h}_{2}^{e}, \ldots, \mathrm{h}_{n}^{e}\right\}$ of the feed-forward network learned in Eq. (1) will be fed into the first layer of HMAM as the input sequence step by step (i.e., $\mathrm{m}^{0}=\mathrm{h}^{e}$ ). Formally, at the i-th time step, the memory slot $\mathrm{m}_{i}^{j}$ at the $j$-th HMAM layer would be updated as:

$$
\mathrm{m}_{i}^{j}=\left\{\begin{array}{cc}
\operatorname{LSTM}\left(\mathrm{m}_{i}^{j-1}, \mathrm{~m}_{i-1}^{j}\right) & i \bmod t^{j}=0 \\
\mathrm{~m}_{i-1}^{j} & \text { else }
\end{array}\right.
$$

where $j \in[1, D] . t^{j}$ is a hyperparameter, and its value is set to be $t^{j}=2^{j-1}$. Hence, we can learn multi-granularity representation $\mathrm{h}_{i}^{c}$ of input token at time step $i$, which is calculated as the concatenation of the output of the $i$-th memory slot at each HMAM layer:

$$
\mathrm{h}_{i}^{c}=\left[\mathrm{m}_{i}^{1}, \mathrm{~m}_{i}^{2}, \ldots, \mathrm{m}_{i}^{D}\right]
$$

To further explore features of the source document, the hidden states of both word-level LSTM and HMAM (i.e., $h_{i}^{e}$ and $h_{i}^{c}$ ) are combined together and fed through another feed forward layer. Formally, the final representation of input token at time step $i$ (i.e., $\mathrm{h}_{i}^{o}$ ) is computed as:

$$
\mathrm{h}_{i}^{o}=\operatorname{ReLU}\left(W_{2}\left[\mathrm{~h}_{i}^{e}, \mathrm{~h}_{i}^{c}\right]+\mathrm{b}_{2}\right)
$$


where $W_{2}$ is a projection parameter and $b_{2}$ is a bias term. The representation of the entire article is $\mathrm{h}^{o}=\left\{\mathrm{h}_{1}^{o}, \mathrm{~h}_{2}^{o}, \ldots, \mathrm{h}_{n}^{o}\right\}$.

\subsection{Dynamic Key-Value Memory-augmented Attention Network}

The dynamic key-value memory-augmented attention network (DMA) module consists of two components: a timely updated keymemory $\mathrm{K} \in \mathbb{R}^{n \times d}$ to keep track of attention history and a fixed value-memory $\mathrm{V} \in \mathbb{R}^{n \times d}$ to store the document features throughout the whole decoding process. Both the key-memory and valuememory consists of $n$ slots indicating the max document length, which are initialized with the document feature vectors $h^{o}$ learned in Eq. (4). At each decoding step, the $i$-th $\operatorname{slot}\left(k_{i} \in \mathbb{R}^{d}\right)$ in keymemory stores the attention status corresponding to the $i$-th document feature vector that is updated along with the decoding process, and the $i$-th slot $\left(v_{i} \in \mathbb{R}^{d}\right)$ in value memory stores the representation of the $i$-th document feature vector $\mathrm{v}_{i}$. In this manner, the model can choose more appropriate salient information. Next, we will introduce the detailed operations of DMA from round $r-1$ to round $r$. To avoid conceptual confusion, we adopt superscripts " $r-1$ " and " $r$ " to represent the variables that are related to the round $r-1$ and round $r$, respectively. Both $\mathrm{K}_{i}^{0}$ and $\mathrm{V}_{i}^{0}$ of the i-th slot before the first decoding step in round 0 are initialized as:

$$
\mathrm{V}_{i}^{0}=\mathrm{K}_{i}^{0}=\mathrm{h}_{i}^{o}
$$

2.2.1 Key-Memory Addressing. At decoding step $t$, we get a "query" vector $\mathrm{q}_{t}$ by taking the concatenation of the hidden state $\mathrm{h}_{t-1}^{d}$ and the previous word embedding $e\left(y_{t-1}\right)$ as input of a LSTM decoder:

$$
\mathrm{q} t=\operatorname{LSTM}\left(\mathrm{h}_{t-1}^{d}, e\left(y_{t-1}\right)\right)
$$

where $\mathrm{q}_{t}$ is used to address from the key-memory. We compute the attention vector $\alpha_{t}^{r}$ over the key-memory $\mathrm{K}_{t}^{r-1}$ as follows:

$$
\begin{gathered}
\alpha_{t, j}^{r}=\operatorname{softmax}\left(u_{t, j}^{r}\right) \\
u_{t, j}^{r}=\mathrm{v}_{\alpha}^{r T} \tanh \left(\mathrm{W}_{\alpha}^{r} \mathrm{q}_{t}+\mathrm{U}_{\alpha}^{r} \mathrm{~K}_{t, j}^{r-1}\right)
\end{gathered}
$$

where $\mathrm{K}_{t, j}^{r-1}$ represents the $j$-th memory slot of the key memory $\mathrm{K}_{t}^{r-1} \cdot \mathrm{W}_{\alpha}^{r}$, $\mathrm{U}_{\alpha}^{r}$, and $\mathrm{v}_{\alpha}^{r}$ are learnable parameters. $\alpha_{t, j}^{r}$ indicates the weight assigned to the $j$-th memory slot $\mathrm{K}_{t, j}^{r-1}$.

2.2.2 Value-Memory Reading. After obtaining the attention weight $\alpha_{t}$, the attentive context document representation $c_{t}$ for generating the $t$-th word is computed by the weighted sum of all slots in the value-memory V. Also, we employ another LSTM (denoted as $L S T M^{\prime}$ ) to imitate the decoder state at round $\mathrm{r}$ and obtain the "intermediate" hidden state $\tilde{h}_{t}^{d}$ with the attentive context vector:

$$
\begin{gathered}
\mathrm{c}_{t}^{r}=\sum_{j=1}^{n} \alpha_{t, j}^{r} \mathrm{~V}_{j} \\
\tilde{h}_{t, r}^{d}=\operatorname{LSTM}^{\prime}\left(\mathrm{q}_{t}, \mathrm{c}_{t}^{r}\right)
\end{gathered}
$$

where $\mathrm{V}_{j}$ is the $j$-th slot in static value-memory and $\tilde{h}_{t, r}^{d}$ is used to update the key memory.

2.2.3 Key-Memory Updating. Inspired by the read-write operations proposed by $[4,11,12]$, the updating process of the keymemory state includes two operations: ERASE and ADD. The ERASE operation decides the content to be removed from the memory state, which is similar to the forget gate in LSTM. With ERASE operation, the model can avoid exploring the same information for multiple times, and therefore avoid generating puzzling words or phrases. Formally, the key-memory state after the erase operation is:

$$
\tilde{\mathrm{K}}_{t, i}^{r}=\mathrm{K}_{t, i}^{r-1}\left(1-\beta_{t, i}^{r} \mathrm{~F}_{t}^{r}\right)
$$

where $\mathrm{F}_{t}^{r}=\operatorname{sigmoid}\left(W_{F}, \tilde{h}_{t, r}^{d}\right)$ and $W_{F}$ is a learnable parameter. $\beta_{t, i}^{r}$ indicates the weight of the $i$-th slot of the memory state, which is similar to that of $\alpha_{t, j}^{r}$ defined in Eq.(7).

The ADD operation decides how much current information (new information) should be added to the visual key-memory state to track the dynamic interaction between the key-memory and the decoder, which is computed as:

$$
\mathrm{K}_{t, i}^{r}=\tilde{\mathrm{K}}_{t, i}^{r}+\beta_{t, i}^{r} \tilde{\mathrm{A}}_{t}^{r}
$$

where $\tilde{\mathrm{A}}_{t}^{r}=\sigma\left(W_{\tilde{A}}, \tilde{h}_{t, r}^{d}\right)$ and $W_{\tilde{A}}$ is a learnable parameter.

\subsection{Summary Decoder}

We use an LSTM decoder to generate the output summary word by word. We initialize the decoder hidden state $\mathrm{h}_{0}^{d}$ with the last item of the learned document representation (i.e., $\mathrm{h}_{0}^{d}=\mathrm{h}_{n}^{o}$ ). The generation probability distribution of the $t$-th word $y_{t}$ is computed over the entire vocabulary:

$$
P_{g}\left(y_{t}\right)=\operatorname{softmax}\left(\mathrm{W}_{o}\left[\mathrm{~h}_{t}^{d} ; \mathrm{c}_{t}\right]\right)
$$

where $W_{o}$ is a trainable parameter. $c_{t}$ is the context vector, which is computed by the value-memory reading operation of DMA defined in Eq.(9) based on the learned attention weight.

To alleviate the out-of-vocabulary (OOV) issue in summary generation, we employ the pointer mechanism to copy tokens from the source document directly. First, we compute the probability $g_{\text {copy }}$ of using the copy mechanism [5] at the decoding step $t$ :

$$
g_{\text {copy }}=\operatorname{sigmoid}\left(\mathrm{W}_{c}\left[\mathrm{~h}_{t}^{d} ; \mathrm{c}_{t}\right]\right)
$$

where $W_{c}$ is a trainable parameter. Then, we obtain the final probability distribution for the output token $y_{t}$ as:

$$
P\left(y_{t}\right)=\left(1-g_{\text {copy }}\right) P_{g}\left(y_{t}\right)+g_{\text {copy }} \sum \alpha_{t, j}
$$

where $\alpha_{t, j}$ represents the dynamic attention weight for the $j$-th token in $X$, which is defined in Eq. (7).

The proposed model is trained in an end-to-end manner by employing the minimum negative log-likelihood estimation. Specifically, the objective is the sum of the negative log likelihood of the target word at each decoding step:

$$
\mathcal{L}=-\sum_{1}^{m} \log \left(P\left(y_{t}\right)\right)
$$

where $m$ denotes the length of the generated summary.

\section{EXPERIMENTAL SETUP}

Datasets. We evaluate the proposed model on two publicly available corpora: CNN/Daily Mail [15] and arXiv [3]. The CNN/Daily Mail dataset is collected by Hermann et al. [6] for reading comprehension task and then modified for abstractive text summarization by Nallapati et al. [15]. It comprises news stories on CNN/Daily Mail websites paired with human-written summaries. In total, there are 287,277 documents for training, 13,368 documents for validation, and 11,490 documents for testing. On average, each document contains 781 tokens. The arXiv dataset is a collection of 215,000 scientific papers from arXiv.org. The average article length is 4938 . 
We use $3 \%$ of the whole dataset for validation, $3 \%$ for testing, and the rest is used for training.

Implementation Details. In the experiments, HMAM contains 3 LSTM layers and the updating timescale for the $i$-th layer is set to be $2^{i-1}$. The hidden size of each LSTM unit is set to be 256 . The number of update times in DMA model is set to be 3. Following [19], we combine the cross-entropy loss with a coverage loss as our overall objective function. The vocabulary size is set to be 50,000 . We use Adam optimizer to train the model with a mini-batch size of 32. During the decoding stage, we adopt the beam-search strategy with beam size of 5 .

Baselines. We evaluate and compare MADY with several state-ofthe-art abstractive text summarization methods, including ABS [14], RAS [2], PGC [19], SUME [13], DeepRL [16], GANsum [9], DisAware [3] and Dual-Enc [22].

\section{EXPERIMENTAL RESULTS}

Main Results. Similar to previous work [15], we evaluate the proposed model using three automatic evaluation metrics (i.e., ROUGE1, ROUGE-2, and ROUGE-L) [8], which are the most widely used automatic metrics for evaluating text summarization systems. In addition, we also use human evaluation to verify MADY by randomly selecting 100 documents from the test sets of both datasets based on their Informativeness (whether the generated summary extracts all salient facets of the source document) and Fluency (whether the generated summary is fluent with a proper grammatical structure). The annotators assign each summary an integer score of 1 (bad), 2 (poor), 3 (not bad), 4 (satisfactory), 5 (good). The experimental results are summarized in Tables 1-2. We can observe that MADY consistently and substantially surpasses the compared models by a noticeable margin on both datasets in terms of automatic evaluation. RAS, SUME and ABS perform poorly because they are incapable of handling OOV words without copy mechanism. Consistent with automatic evaluation results, MADY can generate more informative and fluent summaries than the compared models.

Ablation Study. In order to analyze the contribution of each part to the superiority of MADY, we also report the ablation test in terms of discarding the hierarchical multi-scale abstraction modeling (denoted w/o HMAM) and the dynamic key-value memory-augmented attention model (denoted w/o DMA). The ablation results are summarized in Table 3. As expected, both modules contribute great improvements to MADY. In particular, the performance of MADY decreases sharply when removing the DMA module. This may be explained by the fact that DMA alleviates the problem of generating repetitive words and faultiness summaries. The improvement of HMAM is also significant since it helps the model to learn more comprehensive document representations, which is the basis of the latter summary generation stage.

Case Study. As an intuitive way to show the performance of MADY, we randomly choose one generated summary by MADY and PGC from test data for comparison due to limited space. As shown in Table 4, we observe that our model captures the salient information in different parts of the source article and generates a more specific and meaningful summary.
Table 1: Quantitative results on CNN/Daily Mail.

\begin{tabular}{c|c|c|c|c}
\hline Model & R-1 & R-2 & R-L & Human \\
\hline RAS & 37.46 & 15.11 & 34.45 & - \\
SUME & 37.50 & 14.50 & 33.40 & 2.24 \\
PGC & 39.53 & 17.28 & 36.38 & 2.70 \\
DeepRL & 39.87 & 15.82 & 36.90 & 2.55 \\
GANsum & 39.92 & 17.65 & 36.71 & - \\
MADY & 40.72 & 17.90 & 37.21 & 2.86 \\
\hline
\end{tabular}

Table 2: Quantitative results on ArXiv.

\begin{tabular}{c|c|c|c|c}
\hline Model & R-1 & R-2 & R-L & Human \\
\hline ABS & 29.30 & 8.52 & 27.38 & - \\
Pntr-Gen & 35.14 & 11.02 & 30.92 & 2.70 \\
Dis-Aware & 35.80 & 11.05 & 31.80 & 3.22 \\
Dual-Enc & 36.22 & 11.20 & 32.03 & 3.48 \\
MADY & 37.84 & 11.48 & 33.45 & 3.81 \\
\hline
\end{tabular}

Table 3: Ablation test results on CNN/Daily Mail and arXiv.

\begin{tabular}{c|ccc|ccc}
\hline \multirow{2}{*}{ Model } & \multicolumn{3}{|c|}{ CNN/Daily Mail } & \multicolumn{3}{c}{ arXiv } \\
\cline { 2 - 7 } & R-1 & R-2 & R-L & R-1 & R-2 & R-L \\
\hline MADY & 40.72 & 17.90 & 37.21 & 37.84 & 11.48 & 33.45 \\
w/o HMAM & 40.36 & 17.62 & 36.97 & 37.65 & 11.40 & 33.07 \\
w/o DMA & 39.96 & 17.49 & 36.58 & 35.84 & 11.02 & 31.68 \\
\hline
\end{tabular}

Table 4: Example of a generated summary.

Reference summary: entangling and disentangling capacities are the key manifestation of the nonlocal content of a quantum operation. a lot of effort has been put recently into investigating (dis)entangling capacities of unitary operations, but very little is known about capacities of non-unitary operations. here we investigate (dis)entangling capacities of unital cptp maps acting on two qubits.

Summary generated by PGC: we consider two qubits preserving of positive-qubit unitary for non-local map of two-qubit unitary. we calculate the von neumann entropy of two qubits to realize nonlocal quantum resources in mixed states. in this article we calculate the von neumann entropy of the map of the cnot-class (separated states). the single-shot capacities are presented and different recent results for dis)entangling _ random unitary processes.

Summary generated by MADY: we present the continuous case of the amount of nonunitary single map of the article covers all numerical-qubit unitaries preserving (cptp) exchange maps of the form of single-shot capacities of the cnot-class of entanglement. the structure of the optimal state, and entangling capacities of discreet-mixture which can be decomposed to swap the change of dcnot-mixture. the distillable of the resulting map will be characterized for unitary states. it is found that single-qubit increase (dis)entangling can be used to estimate the amount of entanglement measures of quantum operations, and therefore unitaries preserving two-qubit entanglement can be decomposed on two-qubit processes.

\section{CONCLUSIONS}

In this paper, we propose a novel MADY model for abstractive text summarization with a hierarchical multi-scale abstraction modeling method and dynamic memory by studying how humans comprehend source documents with hierarchical levels of abstraction and write summaries based a dynamic working memory. Experimental results on two benchmark datasets showed that MADY significantly outperforms compared methods.

\section{ACKNOWLEDGMENTS}

This work was partially supported by National Natural Science Foundation of China (No. 61906185), Natural Science Foundation of Guangdong Province of China (No. 2019A1515011705), Youth Innovation Promotion Association of CAS China (No. 2020357), Shenzhen Science and Technology Innovation Program (Grant No. KQTD20190929172835662), Shenzhen Basic Research Foundation (No. JCYJ20200109113441941). 


\section{REFERENCES}

[1] Ziqiang Cao, Wenjie Li, Sujian Li, and Furu Wei. 2018. Retrieve, rerank and rewrite: Soft template based neural summarization. In Proceedings of the 56th Annual Meeting of the Association for Computational Linguistics. 152-161.

[2] Sumit Chopra, Michael Auli, and Alexander M. Rush. 2016. Abstractive Sentence Summarization with Attentive Recurrent Neural Networks. In Proceedings of the 2016 Conference of the North American Chapter of the Association for Computational Linguistics: Human Language Technologies. 93-98.

[3] Arman Cohan, Franck Dernoncourt, Doo Soon Kim, Trung Bui, Seokhwan Kim, Walter Chang, and Nazli Goharian. 2018. A discourse-aware attention model for abstractive summarization of long documents. arXiv preprint arXiv:1804.05685 (2018).

[4] Alex Graves, Greg Wayne, and Ivo Danihelka. 2014. Neural turing machines. arXiv preprint arXiv:1410.5401 (2014).

[5] Jiatao Gu, Zhengdong Lu, Hang Li, and Victor OK Li. 2016. Incorporating copying mechanism in sequence-to-sequence learning. arXiv preprint arXiv:1603.06393 (2016).

[6] Karl Moritz Hermann, Tomas Kocisky, Edward Grefenstette, Lasse Espeholt, Will Kay, Mustafa Suleyman, and Phil Blunsom. 2015. Teaching machines to read and comprehend. In Advances in neural information processing systems. 1693-1701.

[7] Sepp Hochreiter and Jürgen Schmidhuber. 1997. Long short-term memory. Neural computation 9, 8 (1997), 1735-1780.

[8] Chin-Yew Lin. 2004. Rouge: A package for automatic evaluation of summaries. In Text summarization branches out. 74-81.

[9] Linqing Liu, Yao Lu, Min Yang, Qiang Qu, Jia Zhu, and Hongyan Li. 2018. Generative adversarial network for abstractive text summarization. In Thirty-second AAAI conference on artificial intelligence.

[10] Yao Lu, Linqing Liu, Zhile Jiang, Min Yang, and Randy Goebel. 2019. A Multi-task Learning Framework for Abstractive Text Summarization. (2019).

[11] Fandong Meng, Zhengdong Lu, Hang Li, and Qun Liu. 2016. Interactive attention for neural machine translation. arXiv preprint arXiv:1610.05011 (2016).

[12] Fandong Meng, Zhaopeng Tu, Yong Cheng, Haiyang Wu, Junjie Zhai, Yuekui Yang, and Di Wang. 2018. Neural machine translation with key-value memoryaugmented attention. IfCAI (2018)
[13] Ramesh Nallapati, Feifei Zhai, and Bowen Zhou. 2016. Summarunner: A recurren neural network based sequence model for extractive summarization of documents. arXiv preprint arXiv:1611.04230 (2016).

[14] Ramesh Nallapati, Bowen Zhou, Cicero dos Santos, Çağlar Guİłlçehre, and Bing Xiang. 2016. Abstractive Text Summarization using Sequence-to-sequence RNNs and Beyond. In Proceedings of The 20th SIGNLL Conference on Computational Natural Language Learning. 280-290.

[15] Ramesh Nallapati, Bowen Zhou, Caglar Gulcehre, Bing Xiang, et al. 2016. Abstractive text summarization using sequence-to-sequence rnns and beyond. arXiv preprint arXiv:1602.06023 (2016).

[16] Romain Paulus, Caiming Xiong, and Richard Socher. 2017. A deep reinforced model for abstractive summarization. arXiv preprint arXiv:1705.04304 (2017).

[17] Kan Ren, Jiarui Qin, Yuchen Fang, Weinan Zhang, Lei Zheng, Weijie Bian, Guoru Zhou, Jian Xu, Yong Yu, Xiaoqiang Zhu, et al. 2019. Lifelong Sequential Modeling with Personalized Memorization for User Response Prediction. In Proceedings of the 42nd International ACM SIGIR Conference on Research and Development in Information Retrieval. 565-574.

[18] Alexander M Rush, Sumit Chopra, and Jason Weston. 2015. A neural attention model for abstractive sentence summarization. arXiv preprint arXiv:1509.00685 (2015)

[19] Abigail See, Peter J Liu, and Christopher D Manning. 2017. Get to the point: Summarization with pointer-generator networks. arXiv preprint arXiv:1704.04368 (2017).

[20] Louis Shao, Stephan Gouws, Denny Britz, Anna Goldie, Brian Strope, and Ray Kurzweil. 2017. Generating high-quality and informative conversation responses with sequence-to-sequence models. In EMNLP.

[21] Xiaoyu Shen, Yang Zhao, Hui Su, and Dietrich Klakow. 2019. Improving Latent Alignment in Text Summarization by Generalizing the Pointer Generator. In EMNLP-IFCNLP. 3753-3764.

[22] K. Yao, L. Zhang, D. Du, T. Luo, L. Tao, and Y. Wu. 2020. Dual Encoding for Abstractive Text Summarization. IEEE Transactions on Cybernetics 50, 3 (2020), 985-996. 